\title{
Linx
}

Revue des linguistes de l'université Paris X Nanterre

$72 \mid 2015$

Former à l'écrit universitaire, un terrain pour la linguistique?

\section{De la note de lecture à la formulation d'une problématique}

\section{Caroline Scheepers}

\section{OpenEdition}

\section{Journals}

Édition électronique

URL : http://journals.openedition.org/linx/1663

DOI : $10.4000 /$ linx. 1663

ISSN : 2118-9692

Éditeur

Presses universitaires de Paris Nanterre

Édition imprimée

Date de publication : 1 septembre 2015

Pagination : 189-212

ISSN : 0246-8743

Référence électronique

Caroline Scheepers, «De la note de lecture à la formulation d'une problématique », Linx [En ligne] 72 | 2015, mis en ligne le 01 mars 2016, consulté le 02 mai 2019. URL : http://

journals.openedition.org/linx/1663; DOI : 10.4000/linx.1663 


\title{
De la note de lecture à la formulation d'une problématique
}

\author{
Caroline SCHEEPERS \\ Haute École Lucia de Brouckère \& Université catholique de Louvain
}

\begin{abstract}
Résumé
L'initiation à l'écriture scientifique telle qu'elle se décline dans les mémoires de Master peut se focaliser sur plusieurs de ses dimensions, mais la capacité à élaborer une problématique est peut-être l'une des exigences les plus complexes qui se pose au chercheur néophyte. Cette contribution envisage des travaux dans lesquels les étudiants doivent tout à la fois rendre compte d'un ouvrage lu, formuler et étayer un avis critique à son encontre avant de concevoir la problématique qui sera traitée dans leur mémoire. Une analyse sociolinguistique des données révèle trois logiques scripturales contrastées: émergent en effet des discours restitutifs (il s'agit de donner à voir), critiques (il s'agit de faire croire) ou problématisants (il s'agit de faire savoir). La question de la place dévolue à la linguistique, tant en termes de formation que de recherche, sous-tend toute la réflexion.
\end{abstract}

Mots-clés

Problématique, compte rendu, pratiques langagières, indicateurs discursifs.

\section{Introduction}

La présente contribution s'inscrit dans le champ des littéracies et, particulièrement, des littéracies académiques : s'affilier à ce courant suppose une série de parti-pris concomitants. La littéracie est définie par Élisabeth Bautier (2009 : 12) comme « une forme simultanée de pensée, de langue et de langage nécessaires aux apprentissages scolaires et au-delà à l'intégration sociale ». Ainsi, la littéracie englobe tout à la fois des savoirs hétorogènes (sur les fonctions de l'écriture, sur les genres de discours...), des savoir-faire diversifiés (l'utilisation du lexique, de la morphosyntaxe, de l'orthographe...), mais aussi des attitudes, des représentations (le rapport à l'écrit, à l'écriture, à la lecture, à l'apprentissage ; la motivation; l'image de soi comme lecteur et scripteur; la capacité à se percevoir comme capable de rentrer dans la culture de l'écrit, que cette culture soit privée, scolaire ou académique, professionnelle). Dans cette perspective, les dimensions strictement linguistiques (lexique, orthographe, morphosyntaxe) s'articulent à un grand nombre d'autres paramètres : elles ne sont pas isolées et ne font pas l'objet d'une attention exclusive, mais elles ne sont pas pour autant mises sous le boisseau. Elles sont prises dans un réseau complexe et structuré de phénomènes hétérogènes qui, tous, retentissent sur les compétences, les pratiques, les attitudes et les performances des scripteurs.

Comment s'envisage l'apprentissage dans cette conception littéraciée du langage ? La visée est, pour le dire vite, d'acculturer l'apprenant au monde de l'écrit, à ses usages, ses enjeux, ses tensions, ses exigences, ses virtualités, ses possibilités, ses diverses facettes. Par « acculturation », j'entends à la suite de Michel Dabène : « une adaptation progressive, avec ou sans tension, à une nouvelle culture, ici celle du scriptural, caractérisée par un mode particulier d'existence et de fonctionnement de la langue, induisant des formes particulières d'expression, de communication et d'accès au savoir » (1997: 86). Il s'agit d'enrôler les apprenants dans une nouvelle culture, celle de l'écrit ou de certaines formes d'écrit. Dès lors, le propos s'applique tout autant aux jeunes enfants qui entrent en primaire qu'aux étudiants 
universitaires, lesquels maitrisent déjà certaines formes d'écrit, mais moins d'autres, spécifiques au cursus académique ou scientifique. En l'occurrence, le pari est d'initier ces jeunes adultes à la culture de l'écriture scientifique, qui repose sur des codes, des conventions, des savoirs, des habiletés, des représentations spécifiques, parfois a priori très éloignés de leur culture initiale. Autrement dit, le champ des littéracies académiques (entre autres, Pollet, 2001 ; Boch et Grossmann, 2001 ; Rinck, 2012) attribue des spécificités propres à la culture écrite académique. Dès lors, le principe est également d'acculturer les étudiants, fussent-ils de Master, à certains genres discursifs universitaires. Le mémoire en fait partie. Par ailleurs, Élisabeth Bautier (2009) estime que les attentes scolaires, a fortiori académiques, aurait-on envie d'ajouter, vont dans le sens d'une littéracie étendue (full litteracy) : la littéracie étendue suppose des modes de faire, de penser, d'écrire spécifiques, en rupture avec la culture de l'oralité. Dit autrement, il s'agit pour le scripteur de s'emparer de toutes les fonctionnalités que l'écrit permet: l'intériorisation, la distance, la relecture, la ressaisie, la reprise, l'orchestration de voix multiples... Le scripteur, en particulier dans le cadre de son mémoire, doit comprendre qu'il peut s'autoriser à penser, à discuter les livres lus, à interpréter des données, à formuler des questions de recherche, à énoncer des hypothèses, à déterminer des options méthodologiques, à articuler références livresques et données empiriques... Le processus exigé demande du temps, de la lenteur et de la patience, comme le rappelle Caroline Masseron (1997 : 31). Bernard Schneuwly et Joaquim Dolz soulignent également le fait que le temps de l'enseignement n'est pas le temps de l'apprentissage (2006:23). La démarche, par les déplacements très importants qu'elle suppose parfois, peut engendrer chez les étudiants de la frustration, de la violence, des conflits de loyauté : dans certains cas, l'étudiant comprend plus ou moins consciemment que l'université attend de lui qu'il adopte d'autres modes de pensée et de communication que ceux de ses proches.

Le champ des littéracies académiques s'est considérablement étoffé depuis une quinzaine d'années (entre autres, Pollet, 2001; Boch et Grossmann, 2001 ; Rinck, 2012). Plusieurs dimensions propres à l'écrit universitaire ont fait l'objet d'études spécifiques, je pense en particulier à la polyphonie discursive (voir les auteurs précités). Parmi les multiples pratiques cognitives et langagières requises par l'université, celle qui consiste à énoncer une problématique constitue sans doute l'une des plus redoutables. Or, peu d'études ont pris en compte cet aspect particulier. De plus, les définitions d'une problématique peuvent être contradictoires. Pour ma part, je retiens l'acception que lui a conférée Élisabeth Nonnon (2002). Selon cette chercheuse, la problématique de recherche résulte de l'articulation étroite entre des questions de recherche, des hypothèses et des choix méthodologiques. Dans cette perspective, problématiser requiert tout à la fois d'énoncer et de hiérarchiser des questions de recherche, par rapport auxquelles sont avancées des hypothèses puis des orientations méthodologiques susceptibles d'une part d'apporter des éléments de réponses aux questions posées et d'autre part de valider ou d'invalider les suppositions initialement émises. Le terme «problématique » n'est donc guère ici synonyme de "problème ». Le lecteur l'aura compris, problématiser ne va pas de soi et mobilise des compétences de haut niveau, tant sur le plan cognitif que discursif, ce qui tend à légitimer les dispositifs visant à acculturer les étudiants à cette conduite de problématisation.

L'initiation à la recherche s'inscrit, explicitement ou non, au cœur des cours généralement dispensés dans le cadre du Master universitaire, quels qu'ils soient. Si tous les étudiants, une fois diplômés, ne deviendront pas chercheurs, la très grande majorité d'entre eux devra élaborer un mémoire, lequel relève selon Yves Reuter (1998) des discours de recherche en formation. Les exigences liées à ce mémoire sont bien souvent déroutantes pour bon nombre d'étudiants et, depuis Pierre Bourdieu et Jean-Claude Passeron (1964, 1970), nous savons que 
les pratiques discursives universitaires paraissent plus ou moins familières, plus ou moins exotiques, à des étudiants issus d'origines socio-culturelles-économiques parfois très contrastées. Le présent article s'appuie sur des écrits produits par des étudiants de Master dans le cadre d'un cours centré sur les spécificités discursives propres au mémoire. Nous verrons que les dimensions linguistiques s'avèrent centrales : pour les références scientifiques au fondement du cours, pour aider les étudiants à mieux lire les mémoires afin d'en dégager les normes implicites, pour évaluer les copies dans un but normatif, pour étayer l'analyse descriptive et compréhensive des travaux estudiantins, pour esquisser des pistes de remédiation sur la base des constats posés...

\section{Le corpus et son analyse}

Les données empiriques étudiées dans cet article sont issues d'un cours centré sur les éléments discursifs propres au mémoire et destiné à des étudiants de Master 1 en langues et lettres françaises et romanes (Université catholique de Louvain). Le cours (15h), dont je suis titulaire, s'intitule : «Analyse et pratique de l'écrit scientifique : le Mémoire de Master ». Plus concrètement, nous allons nous pencher sur un travail de deux pages imposé aux étudiants. Ce travail est réalisé à domicile et est évalué : la note obtenue compte pour un quart de la note finale. Il est demandé aux étudiants :

1) d'élaborer le compte rendu d'un ouvrage scientifique lu dans le cadre de leur mémoire de Master ${ }^{1}$;

2) d'énoncer et d'étayer un avis critique à propos de ce même ouvrage ;

3) de formuler une première version, toute provisoire et révisable soit-elle, de la problématique qui sous-tendra leur mémoire de master en prenant appui sur le livre lu. Dès lors, il s'agit d'avancer les premières questions de recherche, des tentatives d'hypothèses et des orientations méthodologiques ${ }^{2}$.

Il est bien difficile de catégoriser clairement le genre discursif dans lequel s'inscrit le travail demandé, car il s'apparente en réalité à des genres distincts : il comporte dès lors un net caractère hybride. Ainsi, il a à voir, toutes proportions gardées, avec les notes de lecture que rédigent les chercheurs dans une revue scientifique à propos d'ouvrages publiés par leurs pairs. Le principe est alors de donner au lecteur un aperçu du livre traité (de quoi s'agit-il ?), de formuler un avis critique à son sujet (que peut-on en penser ?), d'argumenter l'avis énoncé (sur quels arguments se fonde mon appréciation ?). À ces tâches déjà redoutables s'ajoute celle de construire la problématique que l'on se propose d'envisager dans le cadre de son mémoire : l'étudiant est tenu d'énoncer des questions de recherche (quelles sont les questions auxquelles mon mémoire est supposé apporter des réponses ?), des hypothèses (quelles tentatives de réponse puis-je avancer à l'entame de ma recherche ?) et des pistes méthodologiques (quelles procédures me permettront de répondre à mes questions et de valider ou d'invalider mes hypothèses ?).

\footnotetext{
${ }^{1}$ L'élaboration du mémoire va s'échelonner au fil des deux années de Master, le mémoire étant déposé en fin de Master 2. Le travail prend place au milieu de l'année de Master 1.

2 Bien sûr, la problématique va fortement évoluer dans les mois qui suivront cette première formulation : les questions vont s'affiner et se hiérarchiser, les hypothèses seront mieux cernées, les choix méthodologiques seront plus précis. Il est probable que la problématique qui sous-tendra in fine le mémoire sera foncièrement différente. Mais je crois indispensable que très vite, dès l'entame du travail, les étudiants s'attachent à problématiser. Faute de quoi, sans savoir ce qu'ils cherchent, au fil de leurs lectures et investigations, les lecteurs risquent de... chercher longtemps, sans trouver grand-chose. Encore trop souvent, les mémoires, dans leur version définitive, donnent à voir une problématique lâche, implicite, incohérente... Dans la conclusion, les étudiants formulent parfois des réponses très intéressantes à des questions qu'ils n'ont pas posées et ne répondent pas à celles qu'ils avaient pourtant avancées.
} 
Les trois quarts restants de la note attribuée à ce cours sont alloués à une épreuve écrite réalisée en auditoire : les étudiants reçoivent un extrait de mémoire rendu anonyme et sont tenus de le décrire et de l'analyser en fonction des indicateurs discursifs de scientificité vus au cours. En d'autres termes, cette introduction respecte-t-elle la structure-type d'une introduction? Le discours est-il adéquatement modalisé ? La polyphonie discursive est-elle négociée de manière pertinente?

Le choix de se centrer exclusivement sur la lecture et l'analyse de discours scientifiques peut paraitre étonnant. En réalité, il se justifie par plusieurs motifs. D'abord, le cours se donne devant un amphithéâtre de soixante-dix étudiants et en tout début de Master 1. Le nombre des apprenants et l'encadrement réduit du cours (je suis seule à intervenir) ne permettent pas d'instituer des ateliers plus pratiques. En outre, puisque le cours se donne dès l'entame du Master 1 , les étudiants sont à ce moment précis très peu voire pas du tout avancés dans l'élaboration de leur mémoire. Certains ont un sujet, d'autres non, certains ont un directeur de mémoire, d'autres pas, certains peinent même à identifier le champ de recherche qui sera le leur. En outre, des séminaires plus méthodologiques sont dispensés par les professeurs dans les domaines de spécialité (linguistique, littérature, didactique). Pour toutes ces raisons, il est complexe d'inviter les étudiants à rédiger une introduction de mémoire, une problématique détaillée, le début du cadre théorique ou un autre texte, quelle qu'en soit la nature.

Dans le travail imposé, sont étroitement articulées des compétences lecturales et scripturales. Sont sollicitées des activités cognitivo-langagières hétérogènes, voire contradictoires : reformuler, évaluer, argumenter et problématiser. Quoi qu'il en soit, la linguistique joue dans le cas présent un rôle à la fois double et central. Premièrement, elle permet d'objectiver des indicateurs discursifs de scientificité ${ }^{3}$ que nous repérons avec les étudiants dans des mémoires anonymisés de manière à mieux cerner les spécificités de ce genre académique particulier. Il s'agit alors de voir comment s'actualisent dans des énoncés authentiques des indicateurs tels que l'inscription du sujet dans son discours (Reuter, 1998), les modalisations (Bronckart, 1996), les indices d'allocution (Rabatel et Grossmann, 2007), la négociation de la polyphonie discursive (Boch \& Grossmann, 2001 ; Rinck, 2012...), la conduite argumentative (Amossy, 2009; Donahue, 2008; Scheepers, 2013...), le lexique (champ lexical, registre langagier...), la morphosyntaxe (valeurs des temps verbaux...), les mécanismes de cohérence et de cohésion du discours (Bronckart, 1996)... La visée se veut alors normative et descriptive: le principe est de mieux cerner le fonctionnement empirique de pratiques langagières complexes, mais aussi d'évaluer les conduites scripturales adoptées par les apprentis-chercheurs. Le cours consiste notamment à familiariser les étudiants aux attentes académiques s'agissant des mémoires (critères d'évaluation...). Une bonne partie du cours consiste à lire des discours de recherche produits par des chercheurs expérimentés ou novices et à en dégager le fonctionnement discursif. Par exemple, un article de Marie-Christine Pollet (2001) fait l'objet d'une analyse et les étudiants sont notamment invités à dégager la problématique qui sous-tend le texte. La tâche s'avère redoutable pour la très grande majorité des étudiants, lesquels peinent à identifier les questions de recherche (surtout quand elles ne sont pas énoncées sous forme interrogative), à hiérarchiser et à restructurer ces mêmes

\footnotetext{
3 J'emprunte la définition de l'indicateur à Madeleine Grawitz : « donnée observable permettant d'appréhender les dimensions, la présence ou l'absence de tel attribut de la réalité étudiée » (2001: 388). Dès lors, les «indices discursifs de scientificité » sont les traits discursifs qui, mis bout à bout, caractérisent l'énoncé scientifique : une certaine manière d'argumenter, de gérer les voix d'autrui, d'articuler théorie et empirie, de modaliser son propos...
} 
questions ou à mettre au jour les principales options méthodologiques adoptées par la chercheuse.

L'essentiel du cours consiste à conduire les étudiants à lire et à décoder les discours scientifiques des chercheurs débutants ou experts pour mieux en révéler les mécanismes implicites. Le pari est donc qu'une meilleure connaissance du genre étudié devrait aider les étudiants à respecter les normes ainsi révélées une fois qu'ils devront prendre la plume et écrire leur propre mémoire. Pour autant, je ne postule pas un transfert automatique entre compétences lecturales et scripturales, mais je m'affilie aux travaux des didacticiens (voir notamment Reuter, 2000) qui ont montré qu'un aller-retour lecture/écriture est susceptible de dévoiler, de démythifier, d'objectiver les mécanismes subtils et complexes de l'écriture de recherche, qu'il s'agisse de la conduite argumentative à adopter ou de la gestion de la polyphonie discursive. On pourrait dire qu'il s'agit d'un apprentissage rédactionnel par imitation d'un modèle.

Deuxièmement, la linguistique me permet de mieux appréhender les tentatives scripturales déployées par les étudiants lorsque munis de ces connaissances sur l'écriture académique, ils doivent s'essayer à rendre compte d'un ouvrage lu, à argumenter leur avis à ce sujet et à formuler une problématique en vue d'élaborer leur propre mémoire. À ce moment, ce sont ces mêmes indicateurs discursifs qui sous-tendent l'analyse des soixante-dix copies reçues. Sont ainsi repérables des pratiques langagières hétérogènes s'agissant de la place réservée à la problématique, de son articulation avec la lecture de l'ouvrage de référence, des actes langagiers mobilisés par le sujet (évaluer, s'autoévaluer, reformuler, interpréter, comparer, questionner...)...

La problématique qui sous-tend la présente contribution peut se décliner en deux principales questions de recherche. La première : quelles pratiques langagières, au sens d'Élisabeth Bautier (1995) ${ }^{4}$, sont repérables dans le corpus constitué ? L'expression «pratiques langagières » n'est pas du tout neutre: je l'emprunte aux travaux d'Élisabeth Bautier, influencés notamment par la sociologie du langage développée par Basil Bernstein (1975). Dans cette perspective, l'analyse des productions d'étudiants ne se subordonne pas à la recherche de manques ou de faiblesses dans les écrits recueillis, mais tente plutôt de prendre en considération ce que les pratiques langagières d'un étudiant signifient de son rapport au monde, au langage, à l'écriture et à l'écrit (Bautier,1998 : 152). Il s'agit de débusquer, au moyen d'une lecture attentive et patiente, les logiques d'écriture qui sous-tendent le discours envisagé : le principe est donc d'identifier comment le scripteur « fait» avec cet exercice qui lui est imposé, comment il l'appréhende, le négocie... Comme le soulignent Dominique Bucheton et Élisabeth Bautier (1997 : 236), l'entrée privilégiée est le texte produit, l'écueil étant de se référer à un texte modèle. Cette dernière démarche ne permettrait pas d'envisager l'activité singulière de production que le scripteur a déployée : or, la focalisation se fait ici davantage sur le travail opéré par le sujet, non sur le texte idéal (donc supposé et reconstruit par le chercheur) qu'il aurait dû énoncer. Le regard du chercheur est donc appelé à se déplacer sur les textes : il doit passer d'une prise en compte des carences, lacunes ou écarts par rapport à une norme à une prise en considération de ce que les étudiants font et savent faire, leurs écrits devant être lus comme "des tentatives pour fabriquer du sens », notent Dominique Bucheton et Élisabeth Bautier (1997 : 230). les analyses habituellement conduites (tant en formation qu'en situation de recherche) foncièrement axées sur la norme, voire sur une sur-

\footnotetext{
${ }^{4}$ Le champ des pratiques langagières ne recoupe donc pas celui des procédures d'écriture, les deux modes d'analyse diffèrent sensiblement.
} 
norme ici académique, s'avèrent à mon avis d'une portée bien limitée et expliqueraient les insuffisances des tentatives didactiques pour travailler les carences ainsi identifiées. En effet, les analyses normatives se révèlent bien souvent binaires : c'est réussi, ça ne l'est pas. Cette vision dichotomique ne prend guère en compte les tentatives effectives, multiples, des scripteurs et leur classement conduit à des typologies duales dans lesquelles il me semble que les textes sont bien à l'étroit. Dans cette typologie binaire se retrouvent en outre associés des textes aux logiques bien hétérogènes. Faute de comprendre les logiques internes qui soustendent les essais de mise en mots des individus, faute d'appréhender véritablement l'épaisseur des textes, le diagnostic mène à des propositions didactiques, hélas, souvent inefficaces car elles s'attachent à des micro-phénomènes textuels qui masquent des logiques bien plus profondes ${ }^{5}$. L'hypothèse générale qui sous-tend les travaux de ce champ est que dans les écrits recueillis se donnent à voir des pratiques différenciées et possiblement différenciatrices, des logiques ou des conduites scripturales distinctes, des postures hétérogènes, des modes diversifiés d'appropriation de la tâche imposée.

La seconde question, plus générale, qui sous-tend la présente contribution est fondée sur les réponses obtenues à la première : quel pourrait être l'apport des sciences du langage pour la recherche et la formation axées sur les littéracies académiques ? D'un point de vue méthodologique, affilier ma démarche au domaine des pratiques langagières revient à entreprendre, en tant que chercheuse, une lecture descriptive et compréhensive des travaux, après avoir conduit, en tant que professeure, une lecture normative et évaluative des productions. Est mis en évidence ce que fait le scripteur, non ce qu'il ne fait pas. L'attention se porte sur les processus de production du scripteur dans sa compréhension-appropriation des règles du genre envisagé, elle porte donc moins sur les textes comme produits finis. Concrètement, comment ai-je procédé ? J'ai appliqué aux étudiants les mêmes indicateurs de scientificité du discours que ceux vus en classe : de la conduite argumentative à la gestion de la polyphonie discursive en passant par la modalisation des propos ou la prise en charge énonciative. Bien entendu, le premier indice discriminant utilisé fut le repérage, le comptage et la répartition des séquences dites reformulatives (quand l'étudiant présente l'ouvrage qu'il a lu), argumentatives (quand l'étudiant discute ce même ouvrage) et problématisantes (lorsque l'étudiant formule la problématique qu'il envisagera dans son mémoire). En définitive, le but sous-jacent à ce décryptage des énoncés est qu'une meilleure connaissance des tentatives empiriques des apprenants permettra à terme d'avancer des propositions de formations plus en prise avec les difficultés réelles des étudiants. Car une fois des conduites scripturales hétérogènes cernées, le regard se fait plus évaluatif et normatif : tous les modes d'écriture recensés se valent-ils eu égard aux attentes académiques?

\footnotetext{
${ }^{5}$ Concrètement, plusieurs genres discursifs ont été étudiés sous l'angle des pratiques langagières qu'ils révèlent. Prenant en compte des copies de philosophie issues d'une classe de nouveaux lycéens de la Seine Saint-Denis, Élisabeth Bautier et Jean-Yves Rochex (1997) caractérisent d'une paraphrase les six types d'écrits qu'ils ont identifiés : «du général au particulier : un enchainement des énoncés qui permet de penser », «écrire, mais ne pas changer », « des mots des autres à ses propres mots : un processus de transformation », « se dire avec les mots des autres », "garder les mots des autres, être objectif, parce que le langage dit le vrai », "lorsque l'écriture scolaire est bloquante». Les mêmes auteurs, aidés de Dominique Bucheton (1997) analysent plus particulièrement la façon dont des élèves de seconde font usage des savoirs enseignés pour élaborer un commentaire composé littéraire. Ils dégagent les conduites suivantes : "l'impossible décollage » (les savoirs enseignés par l'école ne sont pas réinvestis), «l'exploration en franc tireur » (sont convoqués des savoirs autres que les savoirs de l'analyse textuelle), «l'élève appliqué » (lequel restitue les savoirs enseignés), « distance et intégration maximales» (des savoirs divers sont articulés, des savoirs sont construits). Ainsi, ces recherches donnent à voir des «pratiques langagières » distinctes, des logiques ou conduites scripturales hétérogènes, lesquelles donnent à voir des modes différenciés d'appropriation des tâches scripturales prescrites par l'école, le collège ou le lycée.
} 


\section{Trois logiques scripturales contrastées}

La lecture des données suggère trois logiques d'écriture nettement différenciées et différenciatrices. Ces logiques s'opposent quant à un indice particulièrement discriminant dans ce cas, à savoir la place laissée aux énoncés de type reformulatif, argumentatif ou problématisant. Mais au-delà de cette constatation assez sommaire, chaque logique d'écriture voit se corréler des indices ou indicateurs spécifiques. Ce sont bien trois discours qui se laissent appréhender : un discours dit de restitution, sous-tendu par la volonté de «donner à voir », un discours critique, axé sur le souci de «faire croire » et un discours problématisant, visant à «faire savoir ». Je vais détailler et illustrer ci-après chacune de ces logiques.

\subsection{Le discours de restitution : donner à voir}

Il s'agit manifestement pour le scripteur d'en dire le plus possible sur le livre lu : celui-ci fait l'objet d'une présentation extrêmement fouillée. Quant à l'avis critique énoncé par rapport au livre, il se fait très discret, voire presqu'inexistant. Quand une opinion est avancée, il arrive qu'elle ne soit pas du tout étayée par des arguments. De même, la problématique est embryonnaire ou carrément absente. Pour rendre compte de l'ouvrage, l'étudiant recourt majoritairement à la reformulation par rapport à la citation : ainsi, le lecteur ne découvre pas un patchwork plus ou moins habile de citations tissées les unes aux autres. Le scripteur assume généralement l'énonciation en ce sens qu'il paraphrase, résume, décrit, présente, contextualise, introduit, resitue le livre dont il traite. Les éléments axiologiques, quand il y en a, sont sommaires : ils ne suscitent pas de développement argumenté. Le discours se fait essentiellement restitutif. L'extrait suivant permet d'illustrer ces différents phénomènes concomitants. Il est brièvement annoté dans la marge à gauche. 


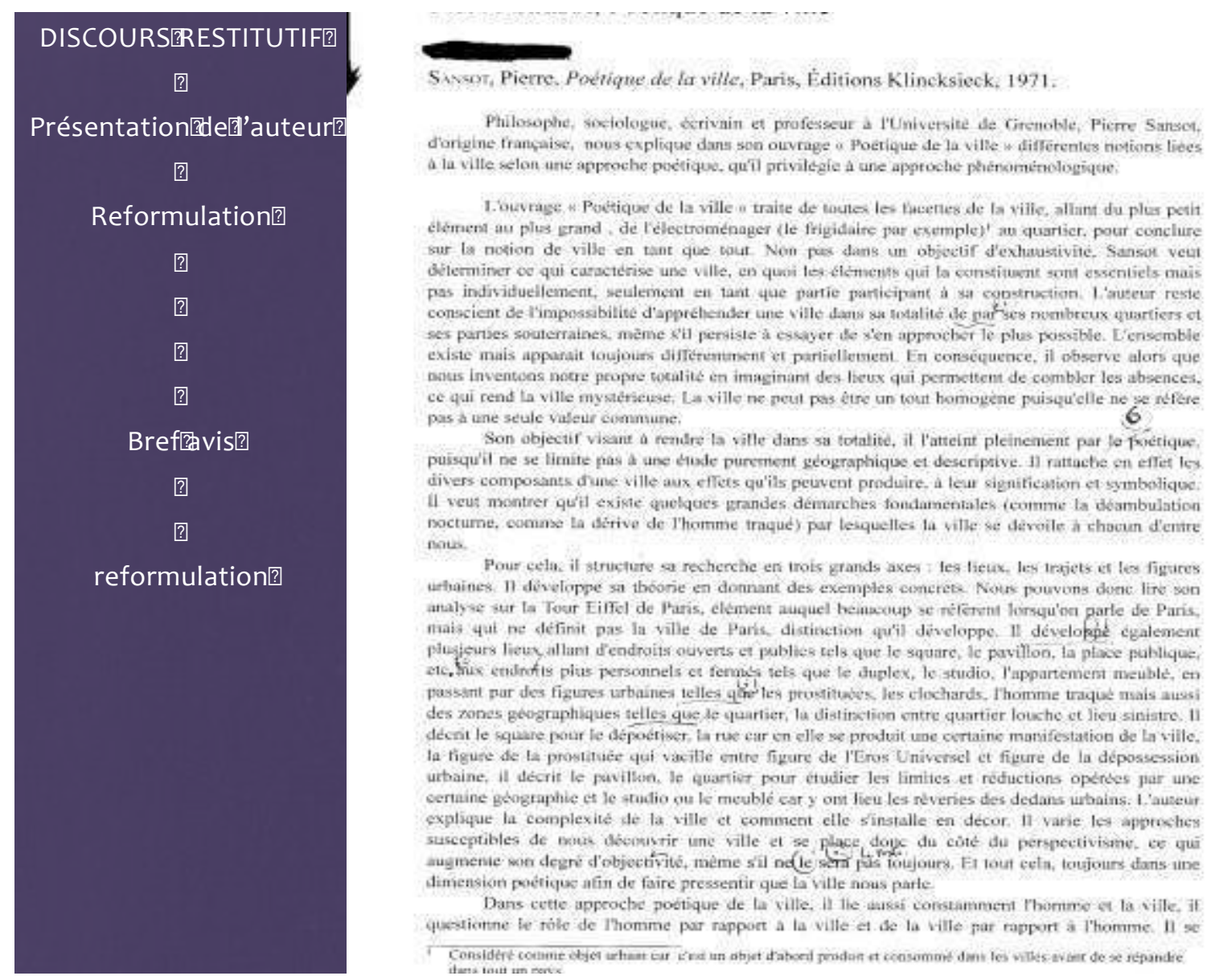

Dans certains cas, il arrive que s'opère une sorte de brouillage énonciatif : le lecteur ne sait plus précisément «qui parle », qui assume le discours. Ainsi, en l'occurrence, à force de reformuler les propos tenus dans le livre sans pour autant insérer des formulations ritualisées du type «toujours selon l'auteur », «le chercheur explique », «l'auteur analyse », l'étudiant devient tout à fait fantomatique, sa présence dans son propre discours s'efface, se dilue. L'extrait qui suit permet d'illustrer ce cas de figure : 


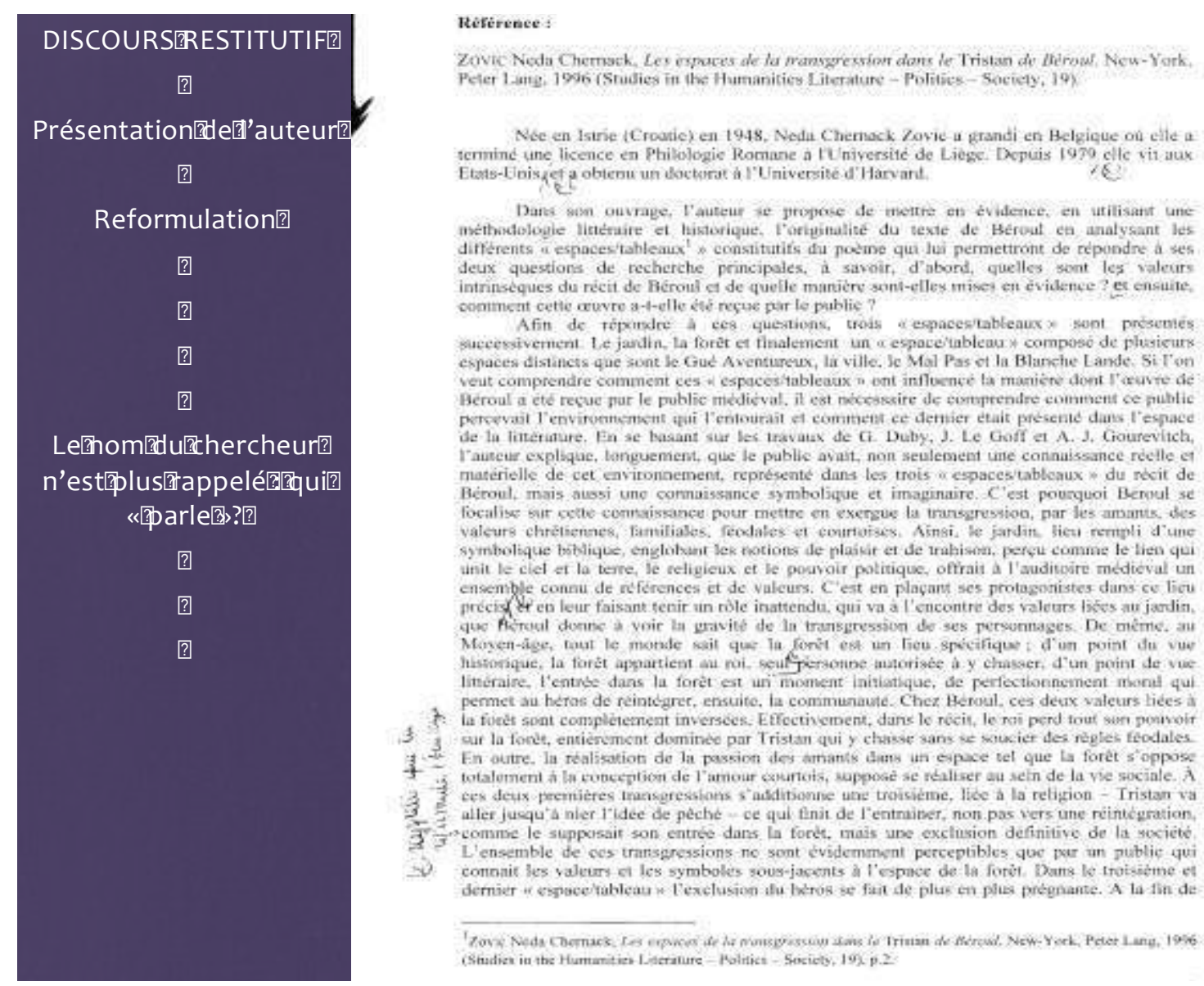

La question qui se pose face à ces discours purement restitutifs est la suivante : quelle est l'origine de cette logique scripturale ? Comment l'expliquer? Les étudiants ont-ils mal compris les consignes, pourtant écrites, qui leur ont été communiquées ? Par facilité, paresse ou conformisme, préfèrent-ils se réfugier derrière la reformulation, laquelle s'avère constituer une prise de risque moindre par rapport à la formulation et à l'étayage d'un avis personnel ou par rapport à la problématisation de la recherche qui va être conduite dans le cadre du mémoire ? Ou les étudiants considèrent-ils qu'ils ne sont pas habilités, en raison précisément de leur statut, à discuter les sources convoquées ? Dès lors, devons-nous attribuer l'absence d'éléments axiologiques à un problème de compétence ou de représentation ? Autrement dit, le souci relève-t-il du vouloir faire ou du savoir faire ? Par ailleurs, pourquoi les étudiants ne problématisent-ils pas ? Peut-être tout bonnement parce qu'ils ne sont guère avancés dans l'élaboration de leur mémoire : peut-être ne sont-ils même pas assurés du sujet auxquels ils désirent se consacrer. Or, l'un des objectifs du travail demandé est d'inviter les étudiants à entamer bien plus tôt leur mémoire et à y consacrer le temps, l'énergie et la réflexion requis, le mémoire se préparant bien en amont de son dépôt. Il est également possible que certains étudiants peinent à tisser des liens entre le livre étudié et leur propre démarche ou qu'ils éprouvent des difficultés à formuler une problématique recevable pour un début de recherche.

\subsection{Le discours critique : faire croire}

C'est une tout autre logique scripturale qui se donne ici à voir. Il s'agit dans le cas présent pour le scripteur de convaincre avant tout le lecteur du bien-fondé de son positionnement quant au livre lu. Deux cas de figure se présentent : soit l'étudiant évalue la pertinence du livre dans l'absolu, soit il en évalue la pertinence par rapport au thème général retenu pour le mémoire, mais la problématique n'est pas formulée en tant que telle. Bref, le scripteur 
reformule, juge et argumente, mais il ne problématise pas (encore ?). Il faut noter par ailleurs que les positionnements adoptés sont souvent nuancés: en particulier, abondent les concessions, introduites de façon stéréotypée par «toutefois» ou «néanmoins ». Il arrive cependant que l'avis émis soit insuffisamment étayé, comme le montre cet extrait: «L'ouvrage de Gérard Quentin nous a apporté beaucoup, à nous, future enseignante. » Mais quel fut exactement cet apport, l'étudiante n'en dit mot. Enfin, il importe de relever l'extrême diversité des arguments convoqués pour appuyer les jugements énoncés : pertinence de la bibliographie, densité du discours, complétude des informations, rigueur, temporalité de la recherche, traitement des données, pertinence de l'introduction ou de la conclusion, structure, méthodologie, honnêteté scientifique, illustrations, exemples, lisibilité... Ainsi, entreprendre de dresser la liste exhaustive des arguments mobilisés s'avère impossible : l'ensemble des indicateurs discursifs de scientificité abordés lors du cours sont mis au service des démonstrations conduites par les étudiants. Bref, faire croire semble bien être la visée sur laquelle se fondent ces discours, même si, pour ce faire, les énoncés de type reformulatif restent bien présents : ils soutiennent la thèse défendue, laquelle, toujours implicite, pourrait être libellée en ces termes, «ce livre est pertinent ou intéressant, ce livre doit être lu » (ou pas). Les énoncés argumentatifs et reformulatifs sont la plupart du temps tissés les uns aux autres avec soin.

L'extrait qui suit montre un étudiant qui discute volontiers ses sources en énonçant différents jugements de valeur et en argumentant ensuite la validité des propos tenus. On le voit, l'avis ainsi constitué s'avère mitigé, l'étudiant n'hésitant pas à pointer les faiblesses qu'il attribue à l'ouvrage, tandis qu'il tempère ces critiques parfois vives par des éloges. Par la suite, l'étudiant évalue la pertinence du livre lu par rapport à sa propre entreprise de recherche. Toutefois, la problématique qui sous-tendra le mémoire n'est qu'esquissée, elle se devine en pointillés, elle n'est guère formulée explicitement et formellement. 

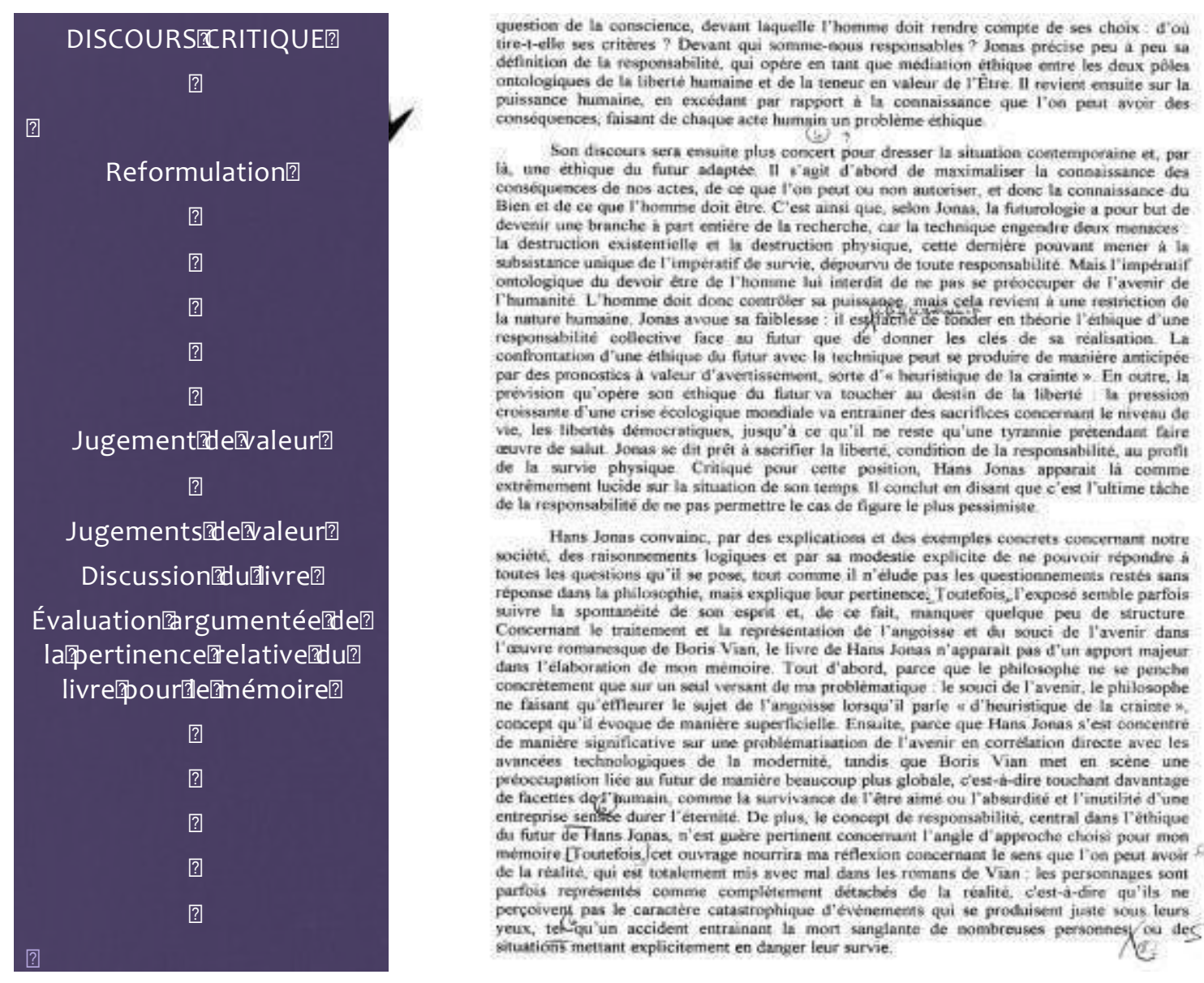

\subsection{Le discours problématisant : faire savoir}

La logique problématisante fait interagir des énoncés reformulatifs, argumentatifs et problématisants : ces trois démarches cognitivo-discursives trouvent à se consolider les unes aux autres. La principale visée poursuivie par ces étudiants consiste à construire et à donner à voir au lecteur la problématique du mémoire en construction. Cela étant, la problématique est présentée sous des formes variées :

- le thème est donné : "Mon mémoire porte sur la reprise des mythes antiques dans les contes de fées du XVIIIe siècle »;

- un questionnement est proposé : «Pourquoi prendre la peine de rassembler ces informations pour en faire une œuvre proprement historique ?»;

- question(s) + hypothèse(s) ;

- question $(s)+$ hypothèse $(s)+$ méthode.

La problématique apparaît de deux façons : soit en bloc, au début ou à la fin du texte, soit le discours est «entrelardé » : reformulation / critique / problématisation sont en interaction permanente. Il arrive que la formulation soit parfois erronée, un étudiant énonçant une hypothèse sous une forme interrogative ou un étudiant avançant une question de recherche au moyen d'une phrase déclarative : "L'hypothèse formulée pour cette partie est celle-ci : Rachilde, aux apparences misogynes, n'est-elle pas plus féministe qu'elle n'y paraît ? » En revanche, dans d'autres cas, la problématique est parfois déjà étonnamment aboutie, alors que nous ne sommes qu'à la moitié de la première année de préparation du mémoire. La problématique est dans certains cas affirmée avec beaucoup d'assurance : «La première partie de mon mémoire consistera 
à passer en revue plusieurs définitions du roman historique pour en proposer ma propre définition ». En outre, l'élaboration du mémoire et/ou de la problématique peut prendre la forme d'un récit (Daunay, 2004), l'étudiant mettant en scène (théâtralisant ?) son processus de recherche.

L'extrait qui suit illustre plusieurs des constats qui viennent d'être faits :
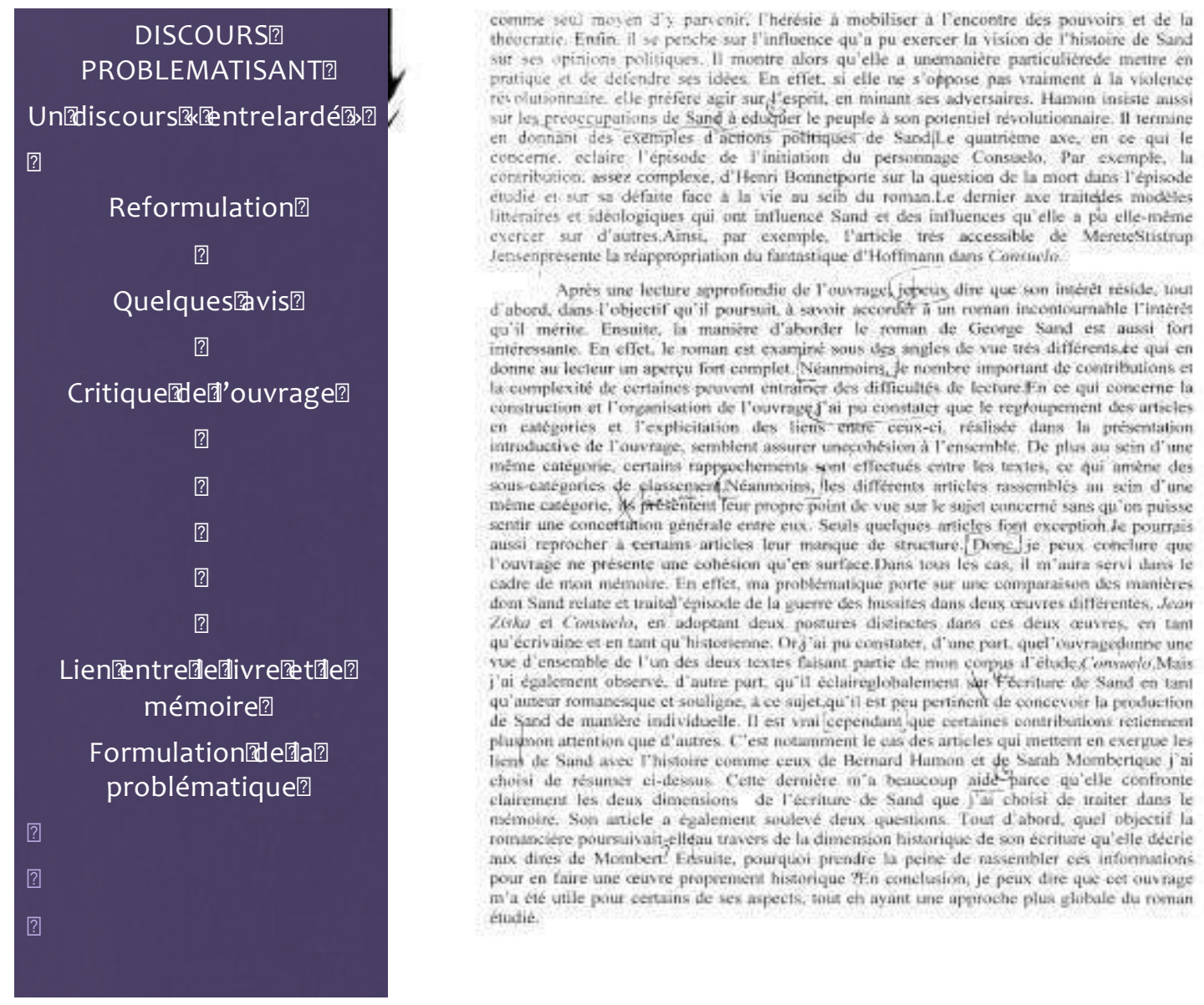

Quant au passage suivant, il révèle une écriture heuristique : non seulement l'étudiante énonce précisément sa problématique, mais en plus elle narre avec soin le cheminement qui a présidé à son émergence progressive. 


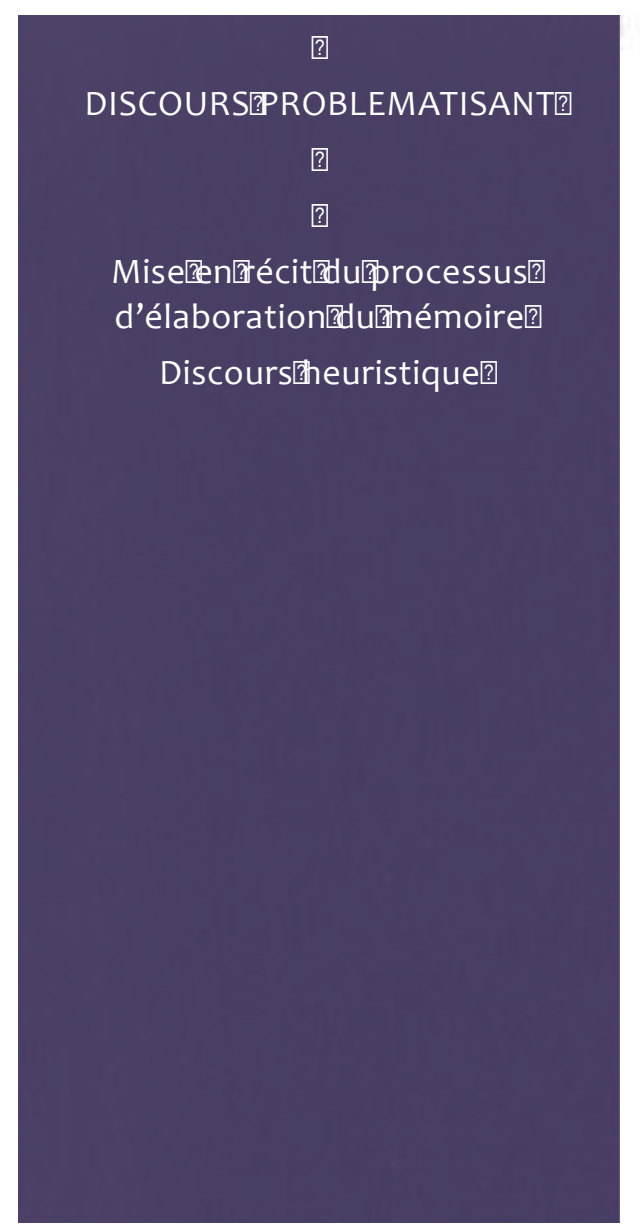

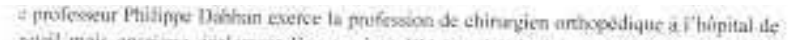

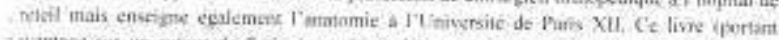

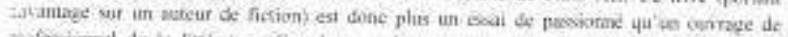

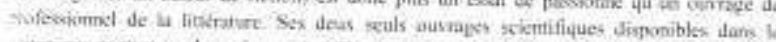

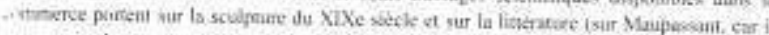

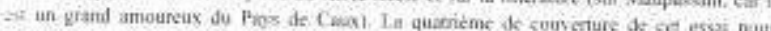

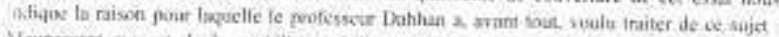

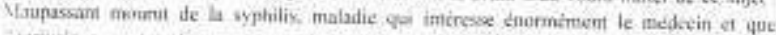

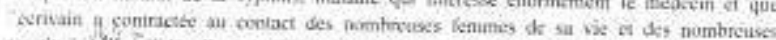

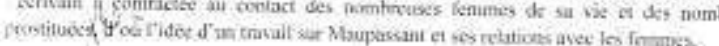

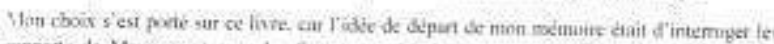

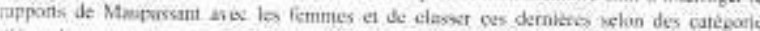

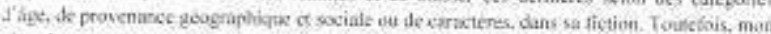

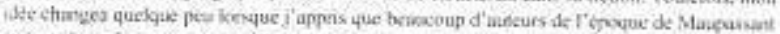

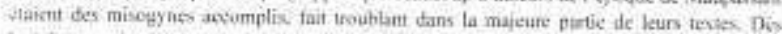

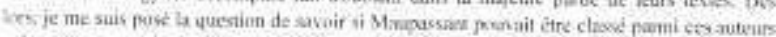

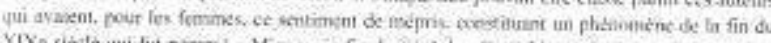

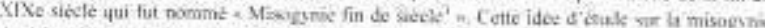

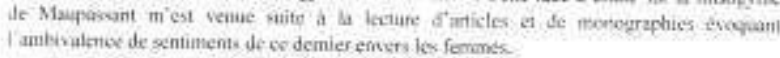

Ce tive ah professeur Dalitan se divise en thes purties principales. separbse en fiten:

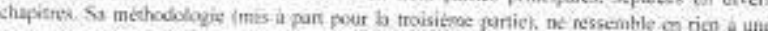

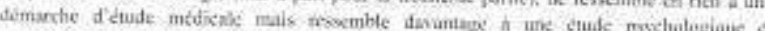

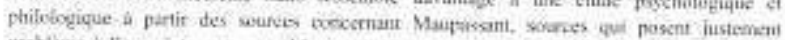

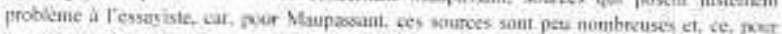

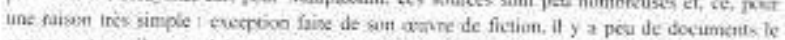

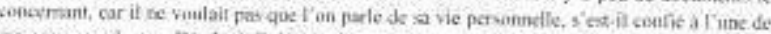
es curnespondantes. Des lors, il decila de facher iu de detruire la plupant de so lestres qu

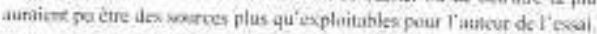

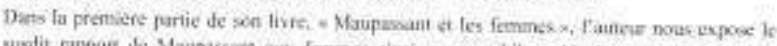

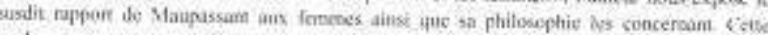

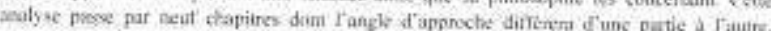

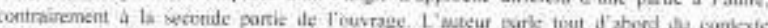

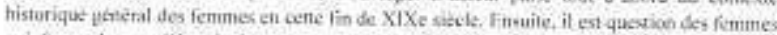

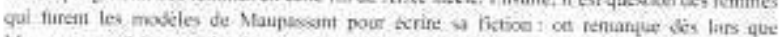

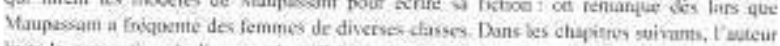

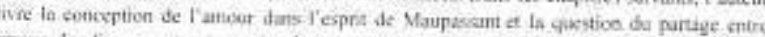

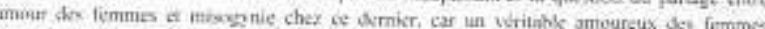

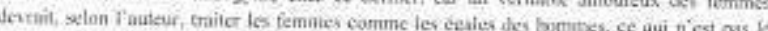

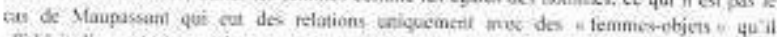

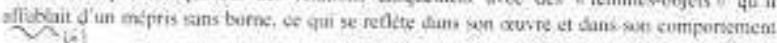
(a)

\section{Conclusion}

J'espère avoir montré que la linguistique joue un rôle central dans le dispositif de rechercheformation que j'ai conçu, puis expérimenté et analysé. Je reprends ci-dessous les différentes fonctions qui ont pu être les siennes, dont l'hétérogénéité des objets traduit finalement l'omniprésence de la description linguistique dans tous les domaines qui nécessitent un commentaire méta-discursif ou langagier :

- Fonder scientifiquement le cours ;

- Repérer avec les étudiants les indicateurs discursifs propres à l'écrit scientifique pour caractériser le genre «compte rendu » ou «mémoire »;

- Inviter ensuite les étudiants à remobiliser ces indicateurs dans leur propre énoncé ;

- Évaluer l'aspect normé du langage ;

- Fonder l'analyse scientifique des discours produits par les étudiants ;

- Mettre au jour des phénomènes non révélés par une lecture normative au moyen d'une lecture descriptive de type sociolinguistique.

La lecture des données a permis de révéler trois logiques scripturales contrastées : une logique reformulative, argumentative et problématisante. Eu égard aux consignes communiquées, c'est bien sûr la troisième qui, sur un plan plus normatif, s'avère la plus aboutie, la plus complexe à manier également. Elle est aussi celle qui suppose la plus grande part de risque, l'auteur devant se positionner, défendre son avis, contreargumenter, définir et articuler questions de recherche, hypothèses et orientation méthodologique. Pourquoi tous les étudiants n'adoptent-ils pas cette conduite 
scripturale, en dépit des consignes pourtant très explicites qui les y invitaient ? La question reste entière. Il faudrait identifier si nous avons affaire à une question d'autorisation, de compétences, d'habiletés, de représentations, de coût cognitif jugé excessif ou d'état d'avancement de la recherche. Pour autant, les exemples épinglés dans cette contribution pourraient soutenir utilement un travail avec les étudiants, travail les amenant à mieux reformuler, mieux argumenter et mieux problématiser, tout en tissant ces trois processus au plus serré.

Toutefois, si je maintiens l'importance, la nécessité même, d'une attention soutenue aux dimensions strictement linguistiques des énoncés, si je plaide pour un recours massif aux sciences du langage par les chercheurs et enseignants, je défends l'idée selon laquelle cette attention et ce recours ne peuvent être exclusifs. En effet, comme le rappelle Élisabeth Bautier (2001 : 118), la catégorie langue est insuffisante pour appréhender la complexité des phénomènes que l'apprenti-scripteur doit prendre en charge pour écrire et des difficultés qu'il éprouve à le faire. De plus, le fait qu'une copie dénote une excellente maitrise des seules dimensions linguistiques peut masquer le fait que l'étudiant est passé à côté de l'objectif du travail, qui demande en l'occurrence d'articuler reformulation, argumentation et problématisation, en se focalisant sur l'un ou l'autre aspect. L'évaluateur pressé peut être ébloui par une certaine virtuosité rhétorique qui masque l'insuffisance du contenu. L'inverse est également vrai : un contenu est jugé insuffisant en raison de sa maladresse de formulation. Une lecture sociolinguistique des copies révèle des phénomènes qu'une lecture normative n'avait pas suffisamment mis en lumière.

Comme Élisabeth Bautier l'ajoute également (2001:132), un enseignement compensatoire portant strictement sur des dimensions linguistiques est sans effet sur les compétences scripturales des apprenants. Non pas qu'ils n'aient pas besoin de connaître les règles du participe passé ou les principaux connecteurs argumentatifs, mais parce qu'écrire suppose bien plus que cela. La chercheuse insiste (Bautier, $2009: 178$ ) : « Il y a rarement transfert de compétence scripturale d'une situation à une autre quand l'acquisition de l'écrit se fait non pas par le biais de la construction d'un sujet pensant et écrivant, mais par celui de l'apprentissage de modèles textuels qui restent extérieurs à l'élève suiviste. » Dès lors, il importe de convoquer les dimensions et outils linguistiques pour les mettre au service de l'émergence, en l'occurrence, d'un apprentichercheur, ce qui suppose dans le même temps l'acquisition de compétences, de pratiques, d'attitudes, de savoirs, de savoir-faire multiples et hétérogènes. D'où la complexité de dimensions imbriquées qui supposent aussi bien une compétence langagière (générale et spécifique) que des savoirs sur les objets convoqués et une représentation claire des buts assignés à l'activité scripturale.

\section{Bibliographie}

AMOSSY, R., 2009, L'argumentation dans le discours, Paris, Armand Colin. BAUTIER, É., 1995, Pratiques langagières, pratiques sociales, Paris, L'Harmattan.

BAUTIER, É., 1998, « Des genres du discours aux pratiques langagières ou des difficultés à penser le sujet social et ses pratiques », dans F. Grossmann (dir.) Pratiques langagières et didactique de l'écrit. Hommage à Michel Dabène, Grenoble, Ellug, p. 145-156.

BAUTIER, É., 2001, «Pratiques langagières, pratiques sociales », Revue française de pédagogie 137, p. 117-161. 
BAUTIER, É., 2009, «Quand le discours pédagogique entrave la construction des usages littéraciés du langage », Pratiques, 143-144, p. 11-26.

BAUTIER, É., ROCHEX, J-Y., 1997, Les nouveaux lycéens. Massification ou démocratisation, Paris, Armand Colin.

BERNSTEIN, B., 1975, Langage et classes sociales, Paris, Minuit.

BOCH, F., GROSSMANN, F. (dir.), 2001, Apprendre à citer le discours d'autrui, Lidil, 24, Grenoble, Ellug.

BOURDIEU, P., PASSERON, J.-C., 1964, Les héritiers, Paris, Minuit.

BOURDIEU P., PASSERON, J.-C., 1970, La reproduction, Paris, Minuit.

BRONCKART, J.-P., 1996. Activité langagière, texte et discours, Lausanne-Paris, Delachaux et Niestlé.

BUCHETON, D., BAUTIER, É., (dir.), 1997, Conduites d'écriture au collège et au lycée professionnel, Versailles, CRDP.

CHARAUDEAU, P., 2008, "L'argumentation dans une problématique de l'influence », Revue Argumentation et analyse $d u$ discours, L'analyse $d u$ discours au prisme de l'argumentation, 1 <http://aad.revues.org/193>

DABÈNE, M., 1997, L'adulte et l'écriture, Bruxelles, De Boeck.

DAUNAY, B,. 2004. «Les mémoires professionnels entre récit et théorie », dans J.-L.

DUFAYS et al. (dir.), Réflexivité et écriture dans la formation des enseignants, Louvain-la-

Neuve, Presses universitaires de Louvain, p. 195-212.

DONAHUE, Chr., 2008, Écrire à l'université, Villeneuve d'Ascq, Presses universitaires du Septentrion.

GALATANU, O., 1998, «Savoirs d'action et représentations de la professionnalité enseignante. Dynamiques argumentatives et effets cognitifs du mémoire professionnel en IUFM », dans F. Cros (dir.), Le mémoire professionnel en formation des enseignants, Paris, L'Harmattan, p. 131-142.

GRAWITZ, M., 2001, Méthodes des sciences sociales, Paris, Dalloz.

KERBRAT-ORECCHIONI, C., 1996, La conversation, Paris, Seuil.

MASSERON, C., 1997, « Pour une didactique de l'argumentation (écrite) : problèmes, objets et propositions I et II », Pratiques 96, p. 7-57.

NONNON, E., 2002, «Formulation de problématiques et mouvements de problématisation dans les textes réflexifs : un point aveugle pour l'enseignant? », Spirale, 29, p. 27-74.

POLLET, M.-Chr., 2001, «Les étudiants et l'écriture de recherche : quelles compétences ? quelles représentations ? quel enseignement?», dans L. Collès et al. (dir.), Didactique des langues romanes, Bruxelles, De Boeck-Larcier, p. 393-398.

RABATEL, A., GROSSMANN, F., (dir.), 2007, « Figures de l'auteur en didactique », Lidil, 35, Grenoble, Ellug.

REUTER, Y., 1998, « De quelques obstacles à l'écriture de recherche », Lidil, 17, p. 12-24.

REUTER, Y., 2000, Enseigner et apprendre à écrire, Paris, ESF.

RINCK, F., 2006, «Gestion de la polyphonie et figure de l'auteur dans les parties théoriques de rapports de stage », Lidil, 34, p. 85-103.

RINCK, F., 2012, «Réflexivité et écrits de recherche. Propositions pour une formation universitaire à et par la littéracie », dans M.C. Pollet (dir.), De la maîtrise du français aux littéracies dans l'enseignement supérieur, Diptyque, Namur, Presses Universitaires de Namur, p. 79-91.

SCHEEPERS, C., 2012, «Soi-même comme un auteur : au cœur des travaux de fin d'études élaborés par de futurs instituteurs en Communauté française de Belgique, des pratiques langagières différenciées », dans M.C. Pollet (dir.), De la maîtrise du français aux littéracies dans l'enseignement supérieur, Diptyque, Namur, Presses Universitaires de Namur, p. 109130. 
SCHEEPERS, C., 2013, L'argumentation écrite, Bruxelles, De Boeck.

SCHNEUWLY, B., DOLZ, J., 2006, Des objets enseignés en classe de français, Rennes, Presses universitaires de Rennes. 